\title{
Forschungsbericht
}

\section{Marcel Lepper \\ Wie kann man Geschichte der Germanistik nach 1945 schreiben?}

\begin{abstract}
This article outlines four ways of writing the history of German Studies after 1945: 1. the history of theory and methods, 2. the history of projects and objects, 3. the history of institutions, 4 . the biographical approach. The discipline is learning how to appreciate polycentric structures and philological traditions which transcend the boundaries of the 19th century and the nation state.
\end{abstract}

Dr. Marcel Lepper: Leiter des Forschungsreferats und der Arbeitsstelle Geschichte der Germanistik, Deutsches Literaturarchiv Marbach, Schillerhöhe 8-10, D-71672, Marbach am Neckar, E-Mail: Marcel.Lepper@dla-marbach.de

„Bitte von der Epochenkante zurücktreten!“, forderte Jürgen Kaube in der Besprechung einer zeithistorischen Antrittsvorlesung an der Humboldt-Universität zu Berlin. ${ }^{1}$ In der Tat ist die Zeitgeschichte den Problemen der Perspektive und des Urteils in verschärfter Form ausgesetzt - dem Spannungsverhältnis zwischen Erinnerung und Archiv, Zeitzeugnis und Investigation, den Reizen und Tücken der Gegenwartsdiagnose. Das gilt, in noch einmal forcierter Weise, für den wissenschaftshistorischen Part, der sich mit der Zeitgeschichte der historischen Fächer auseinandersetzt. Das ,Wie kann ...?` der Titelfrage ist deshalb ernster zu nehmen als es, rhetorisch betrachtet, auf den ersten Blick scheinen mag. Es ist nicht ausgemacht, dass die Hindernisse methodischer, archivarischer, rechtlicher Art überwunden werden können.

An die Stelle der Gelingensgarantie tritt freilich der Praxisbeleg: Seit Jahrzehnten wird Zeitgeschichte der historischen Fächer geschrieben - nicht allein

1 Jürgen Kaube: Ungeduldig - ist Zeitgeschichte möglich? [Besprechung der Antrittsvorlesung von Martin Sabrow: Die Zeit der Zeitgeschichte. Göttingen: Wallstein 2012]. In: FAZ, 6. Juni 2012, S. N3; dem vorliegenden Aufsatz liegen zwei Vorträge zugrunde - der eine gehalten am 25. Juni 2012 im Rahmen der Sommerakademie des Schweizerischen Literaturarchivs und der Universität Zürich im Centre Dürrenmatt in Neuchâtel, der andere am 10. Juli 2012 im Rahmen einer Vortragsreihe an der Universität Bonn; für alle Anregungen danke ich den Teilnehmerinnen und Teilnehmern der Diskussionen. 
für die frühe Nachkriegszeit, sondern auch und längst für eine Phase, die zur Gegenwart in einem unmittelbaren Berührungsverhältnis steht. Das gilt auch und schon rein bibliometrisch betrachtet in beeindruckendem Umfang - für die Geschichte der Germanistik. Aufgrund einer sorgfältigen Sichtung des Materials muss die Titelfrage präzisiert werden. Sie lautet: Wie kann man Geschichte der Germanistik für die Zeit nach 1945 schreiben? Nicht etwa bloß: Wie hat man ,Geschichte der Germanistik seit ihren Anfängen“ in der Zeit seit 1945 beschrieben? In die präzisierte Frage ist die Fachgeschichtsschreibung, sofern sie sich als Teil des Fachs begreift, eingeschlossen. Ebenso ist die Position des ,man“ in der Ausgangsfrage klärungsbedürftig. Hinter dem ,man' steht im vorliegenden Fall kein normatives Verständnis von dem, was ,man tut‘. Vielmehr geht es um die methodische Sichtung der Fülle internationaler Ansätze, die sich mit fachgeschichtlich relevanten Fragen für die Zeit 1945-2011 befassen - und um die Sondierung der Probleme, die sie offen lassen. ${ }^{2}$

1945 ist als politik-, sozial-, kultur- und institutionenhistorische Zäsur in der Fachgeschichtsschreibung geläufig. ${ }^{3}$ Christoph König hat im Internationalen Ger-

2 Exemplarisch Lutz Danneberg/Friedrich Vollhardt (Hg.): Wie international ist die Literaturwissenschaft? Methoden- und Theoriediskussion in den Literaturwissenschaften. Kulturelle Besonderheiten und interkultureller Austausch am Beispiel des Interpretationsproblems (1950-1990). Stuttgart/Weimar: Metzler 1996.

3 Auswahlweise Zdzisław Zygulski: Die polnische Germanistik in den Jahren 1945-1965. In: Annali. Sezione Germanica. Istituto Universitario Orientale di Napoli 9 (1966), S. 189-194; Johannes Hoesle: Die internationale Situation der Germanistik: Tendenzen und Schwerpunkte der italienischen Germanistik seit 1945. In: Colloquia Germanica 8 (1974), S. 95-107; Klaus L. Berghahn: Wortkunst ohne Geschichte: Zur werkimmanenten Methode der Germanistik nach 1945. In: Monatshefte 71 (1979), S. 387-398; Renate Moehrmann: Feministische Ansätze in der Germanistik seit 1945. In: Jahrbuch für internationale Germanistik 11/II (1979), S. 63-84; István Gombocz: Bibliographisches Verzeichnis der germanistischen literaturwissenschaftlichen Publikationen in Ungarn: 1945-1979. In: Acta litteraria Academiae Scientiarum Hungaricae 21 (1979), S. 455-485; Stanislav V. Roznovskij: Das Werk Goethes in den Arbeiten sowjetischer Germanisten: Forschungsbericht für den Zeitraum 1945-1980. In: Goethe-Jahrbuch 98 (1981), S. 131-147; Herbert Ernst Wiegand: Germanistische Wörterbuchforschung nach 1945: Eine einführende Übersicht für Deutschlehrer. In: Der Deutschunterricht: Beiträge zu seiner Praxis und wissenschaftlichen Grundlegung 36/V (1984), S. 10-26; Hans J. Hahn: Kahlschlag und Dschungel in der deutschen Germanistik nach 1945. In: German life and letters: a quarterly review 43/III (1990), S. 246-266; Christoph König (Hg.): Germanistik in Mittel- und Osteuropa 1945-1992. Berlin/New York: de Gruyter 1995; Wilfried Barner/Christoph König (Hg.): Zeitenwechsel. Germanistische Literaturwissenschaft vor und nach 1945. Frankfurt/M.: Fischer 1996; Petra Boden: ,Es geht ums Ganze!': Vergleichende Beobachtungen zur germanistischen Literaturwissenschaft in beiden deutschen Staaten 1945-1989. In: Euphorion 91 (1997), S. 247275; Marcus Gärtner: Kontinuität und Wandel in der neueren deutschen Literaturwissenschaft nach 1945. Bielefeld: Aisthesis 1997; Ursula Amrein: Diskurs der Mitte: antimoderne 
manistenlexikon (IGL) aus guten Gründen das Jahr 1950 als Terminus ante quem gesetzt. Das Jahr erlaubt, die Geschichte des Fachs seit 1800 in die Übergangszone der Nachkriegszeit hinein zu verfolgen:

Der Status der deutschen Germanistik hat sich verändert, allmählich wird man sich überall der Kollegen außerhalb bewußt, wodurch eine internationale Forschungslandschaft erst entsteht. Im deutschen Sprachgebiet geht die Einheit des Fachs verloren. Nicht nur die Wege Westdeutschlands und der DDR trennen sich, sondern auch Österreich entfernt sich, zugunsten einer Austriazistik vor allem des 20. Jahrhunderts, von der gemeinsamen Entwicklung. Gleichzeitig gewinnen die Germanisten in den anderen Ländern, darunter viele Emigranten, an Bedeutung, und es festigen sich die internationalen Verbindungen. ${ }^{4}$

Das Jahr 1950 signalisiert, dass ein näherer Terminus wie etwa 1970 während der Erarbeitungsphase des IGL nicht in Frage kommen konnte - und markiert zugleich die Arbitrarität jeder Zäsur:

Doch das Jahr 1970 kam als Endpunkt des Lexikons nicht in Frage: Wegen der Mengenprobleme, die eine gänzlich andere Konzeption des Lexikons erforderten, wegen der nun fast durchwegs unabgeschlossenen Lebensläufe, vor allem aber wegen der veränderten Stellung der deutschen Germanistik unter den Ländern. Das Jahr 1950 wird zu einem Einschnitt, weil sich in Deutschland vorerst nichts verändert: Man ist frei, die Zäsur kritisch zu setzen, handhabt sie dann aber für einzelne andere Länder gelegentlich flexibel. ${ }^{5}$

Als Terminus ante quem war 1950 richtig gesetzt, als Terminus post quem sprechen gute Gründe für 1945 - gerade um der Erfassung der politischen, wissenschaftlichen, sozialen und kulturellen Übergangszone willen. Der Einschnitt kann für die Fachgeschichtsschreibung, wie andere Zäsuren auch, nur solchen heuristischen Wert haben. Er kann nur angesetzt werden, wenn die komplexen Brüche und Kontinuitäten im theoretischen und methodischen, objekt- und projektbezo-

Dichtungstheorien in der Schweizer Germanistik vor und nach 1945. In: Internationales Archiv für Sozialgeschichte der deutschen Literatur (IASL) 26/I (2001), S. 36-57; Sigurd Paul Scheichl: Germanistik und Politik. Österreich seit 1945 zum Beispiel. In: Geschichte der Germanistik 21/ 22 (2002), S. 33-38; Petra Boden: Grenzschritte: Remigranten in der literaturwissenschaftlichen Germanistik an deutschen Universitäten nach 1945. In: Euphorion 98/IV (2004), S. 425-463; Anna Stroka: Zur Geschichte der Germanistik in Wroctaw (Breslau) in den Jahren 1945-2000. In: Jahrbuch der Schlesischen Friedrich-Wilhelms-Universität zu Breslau 45 (2004), S. 193-206.

4 Christoph König: Einleitung. In: Chr.K.: (Hg.): Internationales Germanistenlexikon (IGL). Berlin/New York: de Gruyter 2003, Bd. 1, S. IX-XXVIII, hier: S. XIII; zu bibliographischen Grundlagen ebd., S. XXI.

5 König: Einleitung (Anm. 4), S. XIV. 
genen, institutionenpolitischen und personengeschichtlichen Sinne berücksichtigt werden.

Zuletzt: Was enthält, was verbirgt der Begriff der Germanistik? Entscheidend ist, dass die Frage nicht so gestellt wird, dass Bereiche der Fachgeschichte von Anfang an ausgeschlossen bleiben. Geschichte der Germanistik kann im genannten Zeitraum nur ein komplexes Gefüge von Einzelbereichen meinen, die durch den Begriff deutlich schwächer integriert sind als das in einer Kernphase der Nationalphilologien der Fall gewesen sein mag. Dies betrifft nicht allein die Drift von Literaturwissenschaft und Sprachwissenschaft innerhalb der neueren Philologien; nicht allein die Entfremdung und wechselseitige Wiederentdeckung von germanistischer Mediävistik, Frühneuzeitforschung und Neuerer deutscher Literaturwissenschaft. ${ }^{6}$ Vielmehr lässt sich die Frage stellen, ob der Fachbegriff seinerseits, wenn man nicht institutionen-, sondern methodengeschichtlich ansetzt, in den vergangenen Jahrzehnten mehr als allein noch heuristischen Charakter hat. Nicht auf das Nachbuchstabieren essentialistisch gestellter Identitätsfrage mag es ankommen, sondern darauf, wie innerhalb der Geschichte der humanities ein aussagekräftiges Untersuchungsszenario umgrenzt werden kann. Kaum eine andere Gruppe geistes- und sozialwissenschaftlicher Disziplinen hat in der Phase nach 1945 stärkeren Theorie- und Methodenimport betrieben, massivere Zentrifugaleffekte gezeigt als die neueren Philologien - und unter ihnen, nicht zuletzt aufgrund institutioneller Gewichtsverhältnisse, in besonderem Maße die germanistische Literaturwissenschaft:

\begin{abstract}
Hier begegnet man einer radikalen Entgrenzung des Text- und Literaturbegriffs, dort einer Rückkehr zum literarischen Text im Zeichen der ,Re-Philologisierung‘. Sowohl die Fragen als auch die Antworten im Hinblick auf die ,Literatur', ihre Voraussetzungen und Funktionen sind heute wohl vielfältiger denn je. Zugleich lassen sich in einer zerklüfteten Forschungslandschaft ganz unterschiedliche interpretive communities (Stanley Fish), kaum aber verbindliche Standards erkennen. ${ }^{7}$
\end{abstract}

Pluralität oder Methodenüberbietungshektik? Reichtum oder Unübersichtlichkeit? Oft genug ausgeblendet bleibt die Kompetenz und arbeitsteilige Spezialisie-

6 Exemplarisch Ursula Peters: Philologie und Texthermeneutik. Aktuelle Forschungsperspektiven der Mediävistik. In: Internationales Archiv für Sozialgeschichte der deutschen Literatur 36/II (2011), S. 251-282; Marcel Lepper/Dirk Werle (Hg.): Entdeckung der Frühen Neuzeit. Konstruktionen einer Epoche der Literatur- und Sprachgeschichte seit 1750. Stuttgart/Leipzig: Hirzel 2011.

7 Matías Martínez/Michael Scheffel: Einleitung. In: M.M./M.S. (Hg.): Klassiker der modernen Literaturtheorie. Von Sigmund Freud bis Judith Butler. München: C.H. Beck 2010, S. 7-10, hier: S. 7. 
rung - für die historische Vielgestaltigkeit von Phänomenen der deutschen Sprache, von deutschsprachigen Literaturen. Ausgeblendet nicht bloß aus irritiertem Selbstbewusstsein, mangelndem Sinn für das Handwerkszeug, sondern aus der Beobachtung heraus, dass damit zugleich zu viel und zu wenig gesagt ist. Germanistik kann, anders als sie im 19. Jahrhundert noch von sich behauptet haben mag, nicht mehr für alles dies zusammen zuständig sein - und muss zugleich mehr erfassen als bloß dies. Bei unterschiedlicher Bewertung bieten die Klagen über den fachlichen Identitätsverlust und die Plädoyers für Binnenpluralität ein strukturell ähnliches Bild, das Michel Espagne zuletzt mit der treffenden Formulierung einer „dezentrierten Germanistik“ aufgegriffen hat. ${ }^{8}$ Die Wissenschaftsgeschichte tut gut daran, nicht identitätsstiftend zu homogenisieren - stattdessen einen ordnenden Zugriff zu wählen, der die fachlichen Subklassifikationen nicht bloß abbildet, sondern die wissenschaftshistorische Grundlage der Philologien, die Erschließung von Traditionspluralität ernst nimmt.

Fachgeschichte ist - in vielen Disziplinen bis heute - Personen- und Institutionengeschichte in jubilatorischem oder schadensabwickelnden Auftrag. Eigenständige Ansätze aus der history of science und den science studies, aus der Wissenschaftstheorie, Wissenschaftssoziologie und Wissenschaftsforschung haben hingegen Zugänge zur Geschichte der humanities gezeigt, die nicht von Fächern als gegebenen Entitäten ausgehen, deren Konturen es bloß mit Fachvertretern zu füllen gilt - sondern von Fragen und Problemen, Methoden und Praktiken, Objektkonstitutionen und Transferbewegungen. Die folgende Gliederung nach 1. Theorie- und Methodengeschichte, 2. Objekt- und Projektgeschichte, 3. Institutionengeschichte, 4. Personengeschichte - hat, wie der Terminus post quem, heuristische Funktion. Sie macht keine Vorgabe, wie Geschichte der Germanistik der jüngsten Zeit geschrieben werden soll, sondern sortiert zunächst die gewählten Zugänge. Aufgreifen kann die Gliederung die Dispositionen, wie jüngere Handbuchunternehmungen sie wählen - etwa das Handbuch Literaturwissenschaft von Thomas Anz. ${ }^{9}$

8 Michel Espagne: Für eine dezentrierte Germanistik. In: Geschichte der Germanistik 39/40 (2011), S. 44-46.

9 Thomas Anz (Hg.): Handbuch Literaturwissenschaft. Stuttgart/Weimar: Metzler 2007.

Bd. 1: Gegenstände und Grundbegriffe; Bd. 2: Methoden und Theorien; Bd. 3: Institutionen und Praxisfelder, in Bd. 3 insb. Ralf Klausnitzer: Institutionalisierung und Modernisierung der Literaturwissenschaft seit dem 19. Jahrhundert, S. 70-147, zur Zeit nach 1945 insbesondere S. 121-147, sowie Dorit Müller: Literaturwissenschaft nach 1968, S. 147-190; überblicksweise auch Jost Hermand:

Geschichte der Germanistik. Reinbek: Rowohlt 1994, S. 114-245; Andreas Gardt: Geschichte der Sprachwissenschaft in Deutschland. Berlin/New York: de Gruyter 1999, S. 332-356. 
Wenn Wissenschaftshistoriker Methodengeschichte betreiben, müssen sie sich nach ihrer eigenen Methodik fragen lassen. ${ }^{10}$ Weisen wissenschaftshistorische Zugänge die Bedingtheit von Frageansätzen und Arbeitsweisen auf, ohne sich selbst aus der Historisierbarkeit zu entlassen, so begegnen sie regelmäßig drei Einwänden: Wissenschaftsgeschichte stecke, erstens, in einem Partizipationsdilemma, das nur die Wahl zwischen fachinterner Selbstlegitimierung oder kritischer Selbstexkommunikation lasse; Wissenschaftsgeschichte sei, zweitens, in der Forschung wie in der Lehre eine Überforderung der ohnehin material- und fragenüberforderten Disziplinen; der Wissenschaftsgeschichte bleibe deshalb, drittens, nur die kommemorative Gelegenheitsarbeit oder die hochspezialisierte Verinselung. Der folgende Überblick begegnet diesen Einwänden, indem er zeigt, welches wissenschaftshistorische Reflexionspotential die Germanistik als großes Fach in den vergangenen Jahrzehnten unter Beweis gestellt hat - aus ihrer philologischen Struktur heraus. Eine solche Fachgeschichte schneidet den Rückweg in die Gegenwart nicht ab - sie verlängert stattdessen produktiv den Weg zum Gegenstand.

\section{Theorie- und Methodengeschichte}

Wer sich der Geschichte der Germanistik seit 1945 nähert, bekommt es mit emphatisch gebrauchten Theorie- und Methodenbegriffen zu tun, die an Modernisierungssemantik und Szientifizierungsprozesse gebunden sind. ${ }^{11}$ Theorie ver-

10 Aus dem genannten Zeitraum Ingrid Strohschneider-Kohrs: Literarische Struktur und geschichtlicher Wandel: Aufriss wissenschaftsgeschichtlicher und methodologischer Probleme. München: Fink 1971; Karl Heinz Metz: Grundformen historiographischen Denkens:

Wissenschaftsgeschichte als Methodologie. München: Fink 1979.

11 Georg Bollenbeck (Hg.): Semantischer Umbau der Geisteswissenschaften nach 1933 und 1945. Heidelberg: Winter 2001, darin u. a. Marcus Gärtner zur Sprache der germanistischen Literaturwissenschaft nach 1945, S. 80-96; Oliver Sill: Kein Ende und ein Anfang. Germanistische Literaturwissenschaft der sechziger und siebziger Jahre. Bielefeld: Aisthesis 2003; Klaus-Michael Bogdal/Oliver Müller (Hg.): Innovation und Modernisierung. Germanistik von 1965 bis 1980. Heidelberg: Synchron 2005; Stefan Scherer: Prägnanz und Evidenz. Philologische Erkenntnis und Verwissenschaftlichung der germanistischen Literaturwissenschaft im disziplinen- und gesellschaftsgeschichtlichen Umbruch der 1950er Jahre. In: Gerhard Kaiser/Matthias Krell (Hg.): Zwischen Resonanz und Eigensinn: Studien zur Geschichte der Sprach- und Literaturwissenschaften im 20. Jahrhundert. Heidelberg: Synchron 2005, S. 33-52; Oliver Sill: Neuer Wein in alten Schläuchen? Anmerkungen zur Literaturwissenschaft zwischen 1965 und 1980. In: ebd., S. 53-70; Julia Kuhlmann: Semantische Umbauten und doppelte Lesarten in Texten der frühen angewandten Sprachwissenschaft in der BRD. In: ebd., S. 169-186; Hans-Harald Müller/Marcel Lepper/ 
spricht, unabhängig von Einzeltheorien und jenseits der allgemeinen Euphorisierung in den literatur- und sprachwissenschaftlichen Fächern, in der Germanistik in besonderer Weise die Immunisierung gegen Ideologien, gegen die Tücken einer diskreditierten Weltanschauungsdisziplin. Dabei bleibt nicht aus, dass Theorieeuphorie, Modernisierungspathos und Innovationsanspruch selbst ideologische Züge annehmen. ${ }^{12}$ Insofern lassen sich, das ist eine erste Beobachtung, Theorie- und Methodenbegriffe historisieren. Sie zeigen Erschöpfungserscheinungen, so dass sich fragen lässt: ,Was hieß Theorie?‘, ohne dass damit behauptet würde, dass jüngere Ansätze ohne theoretische Annahmen und methodische Reflexion auskommen würden - im Gegenteil. ${ }^{13}$ Gewichen sind nicht Theorie und Methode, sondern der emphatische Gebrauch dieser Begriffe. ${ }^{14}$

Zur Historisierbarkeit gehört, zweitens, ein habitueller und rhetorischer Part, der in der klassischen Wissenschaftsgeschichte regelmäßig zu kurz kommt. Wie stark der Theoriebegriff in den Geisteswissenschaften der alten Bundesrepublik

Andreas Gardt (Hg.): Strukturalismus in Deutschland. Literatur- und Sprachwissenschaft. 1910-1975. Göttingen: Wallstein 2010; zur Vorgeschichte des Szientifizierungsprozesses Michael Ansel: Auf dem Weg zur Verwissenschaftlichung der Literaturgeschichtsschreibung. In: Internationales Archiv für Sozialgeschichte der deutschen Literatur 17/I (1992), S. 61-94; zur folgenden Entwicklung Gerd Labroisse: Interpretation als Diskurs: Überlegungen zur Verwissenschaftlichung literaturinterpretativer Aussagen. In: Amsterdamer Beiträge zur neueren Germanistik 23 (1987), S. 155-169; Walter Müller-Seidel: Wissenschaftssprache, Verwissenschaftlichung der Sprache, Sprachkultur: Einleitung zu einer Diskussion. In: Jahrbuch der Deutschen Schillergesellschaft 33 (1989), S. 409-410.

12 Silvio Vietta/Dirk Kemper (Hg.): Germanistik der siebziger Jahre. Zwischen Innovation und Ideologie. München: Fink 2000; Warren Breckman: From the Editor’s Desk. Times of Theory: On Writing the History of French Theory. In: Journal of the History of Ideas 71/III (2010), S. 339-363. 13 Zum polemischen Gebrauch exemplarisch Howard Felperin: Beyond Deconstruction. The Uses and Abuses of Literary Theory. Oxford: Oxford University Press 1988; rekonstruktiv Nikolaus Wegmann: Wer von der Sache nichts versteht, macht Theorie. In: Jörg Schönert (Hg.): Literaturwissenschaft und Wissenschaftsforschung. Stuttgart/Weimar 2000, S. 509-528; aus der gegenwärtigen Diskussion Matthias Buschmeier: Literaturgeschichte nach dem Ende der Theorie? Thesen zu den (Un-)Möglichkeiten einer bedrohten Gattung. Mit Statements von Achim Geisenhanslüke, Ernst Osterkamp, Joseph Vogl. In: Internationales Archiv für Sozialgeschichte der deutschen Literatur 36/II (2011), S. 409-441; einen exzellenten Überblick zur Theoriepolemik seit 1982 gibt Judith Ryan: The Novel After Theory. New York: Columbia University Press 2012, S. 7-17.

14 Marcel Lepper: Wissenschaftsgeschichte als Theoriegeschichte. Ein Arbeitsprogramm. In: Geschichte der Germanistik 29/30 (2006), S. 33-40; M.L.: Theoriegenerationen 1945-1989. In: Zeitschrift für Germanistik N.F. 18/II (2008), S. 244-249; M.L.: Was war Theorie? (Rezension zu Matías Martínez/Michael Scheffel (Hg.): Klassiker der modernen Literaturtheorie. Von Sigmund Freud bis Judith Butler. München: C.H. Beck 2010). In: Journal of Literary Theory (JLTonline), 4. Januar 2012 [http://nbn-resolving.de/urn:nbn:de:0222-001946]. 
mit provokativen Gesten und lebensstilistischen Entscheidungen verbunden war, weisen u. a. Philipp Felsch und Ingo Berensmeyer nach. ${ }^{15}$ Schließlich zeigen sich, drittens, komplizierte Überlagerungen und Transferbewegungen, die sich gegen die Behauptung blockförmiger Theorie- und Methodenkontinua sperren. ${ }^{16}$ Der vielgescholtene Theorie- und Methodeneklektizismus muss wissenschaftshistorisch als eigenes, komplexes Phänomen in seinen historischen Grenzen begriffen werden. ${ }^{17}$

Ein Spezifikum, das in klassischen Überblicksdarstellungen häufig verschliffen wird, muss in der Geschichte der Germanistik besondere Aufmerksamkeit auf sich ziehen: Das Fach zeichnet sich, intensiv mit seiner politischen Selbsthistorisierung beschäftigt, nach 1945 durch ein überdurchschnittliches Maß an Außenreferenz aus. Theorie- und methodenpolitische Stichwortgeber für germanistische Vorhaben finden sich vielfach außerhalb des germanistischen Fachhorizonts in den Nachbarphilologien, in besonderer Weise in der Anglistik, Romanistik und Slawistik, ebenso in der Philosophie, der Soziologie, der Psychologie, den Kunstund Medienwissenschaften. ${ }^{18}$ Zwar zeigen Germanisten wie Günther Müller (1890-1957), Paul Böckmann (1899-1987) und Wolfgang Kayser (1906-1960) ein ausgeprägtes technisches und formales Interesse, das einer folgenden Generation den theoretischen und methodischen Szientifizierungsschritt erleichtert. ${ }^{19}$ Fragt

15 Philipp Felsch: Merve oder Was war Theorie? Hamburg: Philo Fine Arts [erscheint 2012]; Ingo Berensmeyer: Gestus und Geltung. Zur Rhetorik der Theorie. In: Deutsche Vierteljahrsschrift für Literaturwissenschaft und Geistesgeschichte 75/III (2001), S. 491-536.

16 Früh differenziert bei Hans-Harald Müller: Tendenzen der westdeutschen Literaturwissenschaft nach 1965. Dargestellt an den Antworten auf die Probleme einer wissenschaftlichen Textinterpretation. In: Sprache und Literatur in Wissenschaft und Unterricht 15 (1984), S. 87-114.

17 Karlheinz Fingerhut: Intelligenter Eklektizismus: Über die fachdidaktische Anwendung literaturwissenschaftlicher Methoden. In: Der Deutschunterricht 46/V (1994), S. 32-47.

18 Exemplarisch zeigen lässt sich das rezeptive Verhältnis zu den Nachbarphilologien an der anglistisch und romanistisch geprägten Rezeptionstheorie in der Bundesrepublik und in der DDR; entsprechend Mandy Funke: Rezeptionstheorie, Rezeptionsästhetik. Betrachtungen eines deutsch-deutschen Diskurses. Bielefeld: Aisthesis 2004; Wolfgang Adam u. a. (Hg.): Wissenschaft und Systemveränderung: Rezeptionsforschung in Ost und West - eine konvergente Entwicklung? Heidelberg: Winter 2003; Dorothee Kimmich/Bernd Stiegler (Hg.): Zur Rezeption der Rezeptionstheorie. Berlin: Berliner Wissenschaftsverlag 2003.

19 Hans-Jürgen Schings: Zum hundertsten Geburtstag von Paul Böckmann (04. 11. 189922. 04. 1987). Rede, gehalten am 3. November 1999 im Deutschen Literaturarchiv Marbach. In: Jahrbuch der Deutschen Schillergesellschaft 44 (2000), S. 277-290; Orlando Grossegesse/ Erwin Koller (Hg.): Literaturtheorie am Ende? 50 Jahre Wolfgang Kaysers ,Sprachliches Kunstwerk'. Internationales Kolloquium 8.-9. Oktober 1998, Braga, Portugal. Tübingen/Basel: Francke 2001; Eberhard Lämmert: Strukturale Typologien in der Literaturwissenschaft zwischen 1945 und 1960. In: Hans-Harald Müller/Marcel Lepper/Andreas Gardt (Hg.): Strukturalismus in 
man aber nach international strahlkräftigen, theoretischen und methodischen Eigenleistungen der Nachkriegsgermanistik, so bleibt man versucht, auf so eigenständige wie, institutionenpolitisch betrachtet, unrepräsentative Theoretiker wie Käte Hamburger (1896-1992) und Peter Szondi (1929-1971) zu verweisen. ${ }^{20}$

An die verstärkte Außenreferenz der Germanistik nach 1945 ist ein erhöhtes Maß an historischer Selbstreferenz gekoppelt. Zeichnen sich die Philologien seit ihren Anfängen, nicht erst seit einer im späten 19. Jahrhundert einsetzenden Phase forcierter Selbstkritik gegenüber anderen Fächern durch ein überdurchschnittliches Maß an historischer Selbstbeschreibung aus, so muss die Geschichte der Germanistik nach 1945 mit einem noch einmal erhöhten Anteil an Selbstevaluation rechnen. Das Eingeständnis eines verzögerten Methodenwandels in der Germanistik ist in fachlichen Selbstbeschreibungen an die Eroberung der wissenschaftshistorischen Reflexion gebunden - und an Gedächtnisorte wie den Münchner Germanistentag 1966. ${ }^{21}$ Mit der Einrichtung der Marbacher Arbeitsstelle für die Erforschung der Geschichte der Germanistik 1972, die 1986 unter der Leitung von Christoph König ihre Tätigkeit im eigentlichen Sinne aufnimmt, gewinnt die Germanistik einen Ort der systematischen Materialarbeit und der historischen Reflexion. ${ }^{22}$ Zur Selbstbeschreibung gehört auch die umfangreiche

Deutschland. Literatur- und Sprachwissenschaft 1910-1975. Göttingen: Wallstein 2010, S. 229-272.

20 Jörg Paulus: Theoretische Philologie: Annäherung an eine disziplinäre und methodische Leerstelle. In: Germanisch-romanische Monatsschrift N.F. 59/I (2009), S. 33-50; Jean Bollack: Zukunft im Vergangenen. Peter Szondis materiale Hermeneutik. In: Deutsche Vierteljahrsschrift für Literaturwissenschaft und Geistesgeschichte 64 (1990), S. 370-390; Stefan Scherer: Philologische Modernisierung in der Restauration. Literaturwissenschaft in den 1950er Jahren: Peter Szondi. In: Jörg Schönert (Hg.): Literaturwissenschaft und Wissenschaftsforschung. Stuttgart/Weimar 2000, S. 292-316; Johanna Bossinade (Hg.): Käte Hamburger: zur Aktualität einer Klassikerin. Göttingen: Wallstein 2003; Marcel Lepper: Genau und anders: Zum Nachlass von Käte Hamburger. In: Zeitschrift für Germanistik N.F. 18/III (2008), S. 734-738.

21 König: Einleitung (Anm. 4), S. XIV; Eberhard Lämmert: Germanistik - eine deutsche Wissenschaft. In: Benno von Wiese/Rudolph Henß (Hg.): Nationalismus in Germanistik und Dichtung. Dokumentation des Germanistentages in München vom 17.-22. Okt. 1966. Berlin: Erich Schmidt 1967, S. 15-36; Karl Otto Conrady: Deutsche Literaturwissenschaft und drittes Reich. In: ebd., S. 37-60; Eberhard Lämmert: Germanistik - eine deutsche Wissenschaft. In: Universitätstage 1966. Veröffentlichung der Freien Universität Berlin. Ausgabe:

Nationalsozialismus und die deutsche Universität. Berlin: de Gruyter, S. 76-91; E.L. u. a.: Germanistik - eine deutsche Wissenschaft [1967]. 6. Aufl. Frankfurt/M.: Suhrkamp 1980; rückblickend auf 1966 Jörg Schönert: ,Stuttgart 72‘ - zu einem historischen Germanistentag in Verantwortung von Walter Müller-Seidel [2012]. In: http://www.walter-mueller-seidel.de/ materialien.php (Stand: 2. Juli 2012), S. 1-2.

22 Walter Müller-Seidel: Geschichte der Germanistik. Zur Begründung einer Arbeitsstelle in Marbach am 14. April 1972. In: Jahrbuch der Deutschen Schillergesellschaft 17 (1973), 
,Wozu?'-Literatur, die auf externen Legitimationsdruck reagiert. ${ }^{23}$ Werkstattaufzeichnungen, Memoiren und Briefausgaben ergänzen das Gattungstableau. ${ }^{24}$ Die Verengung des Theorie- und Methodenwandels auf ideologiekritische und universitätspolitische Fragen der bundesrepublikanischen Germanistik verstellt freilich den internationalen Horizont: 1966 ist nicht bloß das Jahr des Münchner Germanistentags, sondern auch das Jahr der Strukturalistenkonferenz von Baltimore. ${ }^{25}$ Es ist das Jahr, in dem Peter Szondi eine Reihe prominenter Literaturwissenschaftler, Philosophen und Soziologen an die Freie Universität Berlin einlädt -

S. 584-588; Christoph König: Fachgeschichte im deutschen Literaturarchiv. Programm und erste Ergebnisse. In: Jahrbuch der Deutschen Schillergesellschaft 29 (1985), S. 377-405; Marcel Lepper: Wissenschaftsgeschichte im Deutschen Literaturarchiv Marbach. In: Mitteilungen des Deutschen Germanistenverbandes 53/I (2006), S. 110-121.

23 Siegfried Unseld (Hg.): Wie, warum und zu welchem Ende wurde ich Literaturhistoriker: eine Sammlung von Aufsätzen aus Anlaß des 70. Geburtstags von Robert Minder. Frankfurt/M.: Suhrkamp 1972, mit Beiträgen von Richard Alewyn, Roger Bauer, Pierre Bertaux, Karl Otto Conrady, Leonard Forster, Reinhold Grimm, Rainer Gruenter, Henry Hatfield, Walter Jens, Walther Killy, Volker Klotz, Eberhard Lämmert, Victor Lange, Franz H. Mautner, Peter Wapnewski, Kurt Wölfel; Wolfgang Prinz/Peter Weingart (Hg.): Die sog.

Geisteswissenschaften - Innenansichten. Frankfurt/M.: Suhrkamp 1990, darin die Beiträge von Wolf-Dieter Stempel, Eberhard Lämmert, Wilfried Barner und Wilhelm Voßkamp zur Sprach- und Literaturwissenschaft nach 1945 (S. 161-203; S. 240-247); Peter Weingart/Wolfgang Prinz u. a. (Hg.): Die sog. Geisteswissenschaften - Außenansichten. Frankfurt/M.: Suhrkamp 1991; darin insb. zur germanistischen Fachentwicklung, zu Publikationsmustern und Denominationen in der Facheinheit (S. 156-180; S. 278-323; S. 386-397); Eberhard Lämmert: Das überdachte Labyrinth. Ortsbestimmungen der Literaturwissenschaft 1960-1990. Stuttgart: Metzler 1991; Helmut Arntzen: Unsinn und Sinn der Germanistik. Weinheim: Beltz 1996; Jürgen Wertheimer: Wozu Germanistik? In: Florian Keisinger u. a. (Hg.): Wozu Geisteswissenschaften? Kontroverse Argumente für eine überfällige Debatte. Frankfurt/M./New York 2003, S. 131-135; Jan Erik, Antonsen/Maria-Christina Boerner/Sabine Haupt/Reto Sorg (Hg.): Was heißt und zu welchem Ende studiert man Literaturwissenschaft? Festschrift für Stefan Bodo Würffel zum 65. Geburtstag. München: Fink 2009.

24 Darunter Arbeitsnotizen wie bei Klaus Briegleb: Literatur und Fahndung: 1978 - ein Jahr Literaturwissenschaft konkret. Aufzeichnungen. München: Hanser 1979; Erinnerungen wie bei Egon Schwarz: Keine Zeit für Eichendorff. Chronik unfreiwilliger Wanderjahre. Königstein/Ts.: Athenäum 1979; Peter Wapnewski: Mit dem anderen Auge. Erinnerungen 1922-1959. Berlin: Berlin Verlag 2005; Briefausgaben wie bei Peter Szondi: Briefe. Hg. v. Christoph König/Thomas Sparr [1993]. 2. Aufl. Frankfurt/M.: Suhrkamp 1994; Hans Mayer: Briefe 1948-1963. Hrsg. v. Mark Lehmstedt. Leipzig 2006.

25 Richard Macksey/Eugenio Donato (Hg.): The Structuralist Controversy: The Languages of Criticism and the Sciences of Man [1970]. 2. Aufl. Baltimore: Johns Hopkins UP, 1972; Marcel Lepper: Die strukturalistische Kontroverse, die keine war. Die Konferenz von Baltimore 1966 und die Folgen. In: Ralf Klausnitzer/Carlos Spoerhase (Hg.): Kontroversen in der Literaturtheorie. Bern: Lang 2007, S. 311-326 (Publikationen zur Zeitschrift für Germanistik; N. F., 19); M.L.: Strukturalismus: ein frühes und ein spätes Ende. In: M.L./Hans-Harald Müller/ 
darunter Jean Starobinski, Paul de Man, Jean Bollack, Pierre Bourdieu, Jacques Derrida, Geoffrey Hartman, Peter Demetz, Hans Robert Jauß, René Wellek, Theodor W. Adorno, Harald Weinrich und Gershom Scholem. ${ }^{26}$

Theorie- und Methodengeschichte zeigt, für die Germanistik nach 1945 spezifiziert, evaluative Ergebnisse, die für eine kontinuierlich gedachte Legitimationshistorie irritierend sein mögen. In den Klassikern der Literaturtheorie versammelt der Germanist Horst Turk 1979 Positionen von Boileau bis Barthes - und kommt damit zu Recht dem Nachholbedarf eines großen Fachs entgegen. Dass Friedrich Kittler den Nietzsche-Artikel schreibt, hat in sich fachgeschichtlichen Reiz - für die Zeit nach 1945 bietet der Band freilich nur Croce, Lukács, Mukařovský, Jakobson und Barthes. ${ }^{27}$ Unter den Texten zur Literaturtheorie der Gegenwart, herausgegeben von Dorothee Kimmich, Rolf Günter Renner und Bernd Stiegler, verweisen in der ersten Auflage (1996) allein die Positionen von Siegfried J. Schmidt (geb. 1940) und Friedrich Kittler (1943-2011) auf germanistische Provenienz nach 1945, in der aktualisierten Neuausgabe (2008) tritt Albrecht Koschorke (geb. 1958) hinzu. ${ }^{28}$ Bei Matías Martínez und Michael Scheffel in den Klassikern der modernen Literaturtheorie gehört von 18 Kapiteln ein einziges einer Germanistin - nämlich Michael Scheffels Beitrag zu Käte Hamburger. ${ }^{29}$ Was für die Literaturwissenschaften gilt, lässt sich, mutatis mutandis, auch in der Sprachwissenschaften feststellen: Theorie- und Methodengeschichte nach 1945 lässt sich nur noch international und komparativ rekonstruieren. ${ }^{30}$ Umgekehrt erbringt die Methodengeschichte aus germanistischer Perspektive eine Leistung für die Nachbardisziplinen - so Jost Schneiders Methodengeschichte der Germanistik, deren Lemmata sich in ihrer Relevanz nicht auf die Neuere deutsche Literaturwissenschaft beschränken. ${ }^{31}$

Andreas Gardt (Hg.): Strukturalismus in Deutschland. Literatur- und Sprachwissenschaft 1910-1975. Göttingen: Wallstein 2010, S. 357-370.

26 Christoph König, unter Mitarbeit von Andreas Isenschmid: Engführungen: Peter Szondi und die Literatur. Marbach 2004, S. 76-77; 109; Anna Kinder/Marcel Lepper: Peter Szondis Einführung zu Geoffrey Hartman: Strukturalismus und Literaturwissenschaft (1966). In: Geschichte der Germanistik 31/32 (2007), S. 105-108.

27 Horst Turk (Hg.): Klassiker der Literaturtheorie. Von Boileau bis Barthes. München:

C.H. Beck 1979.

28 Dorothee Kimmich/Rolf Günter Renner/Bernd Stiegler (Hg.): Texte zur Literaturtheorie der Gegenwart [1996]. Vollst. überarb. und akt. Neuausgabe. Stuttgart: Reclam 2008.

29 Martínez/Scheffel: Einleitung (Anm. 7), S. 148-167.

30 Ulrike Haß-Zumkehr/Christoph König (Hg.): Literaturwissenschaft und Linguistik von 1960 bis heute. Göttingen: Wallstein 2003; Christoph König (Hg.): Das Potential europäischer Philologien: Geschichte, Leistung, Funktion. Göttingen: Wallstein 2009.

31 Jost Schneider (Hg.): Methodengeschichte der Germanistik. Unter redaktioneller Mitarbeit von Regina Grundmann. Berlin/New York: de Gruyter 2009; zur Begriffsarbeit die ausführliche Besprechung von Christina Riesenweber. Zur Methodengeschichte der Germanistik. In: 
Wenn der Blick auf eine Einzelphilologie wie die Germanistik gelenkt wird, fällt auf, dass weder eine bloße Theorierezeptionsgeschichte noch eine Theoriewiderstandsgeschichte, weder eine Import- noch eine Mangelgeschichte den Punkt trifft. Vielmehr wird deutlich, dass die Leistungsgrenzen der Nationalphilologien erreicht sind, wenn sich theoretische und methodische Prägungen quer zu den Fachzuordnungen verhalten. Theorie- und methodengeschichtliche Analysen nutzen längst das Potential der Digital Humanities, wenn es gilt, große, transdisziplinäre Korpora zu bewältigen. ${ }^{32}$ Als heuristische Vorgabe kann die germanistische Fachzuordnung helfen, ein Korpus einzugrenzen, wenn es gilt, historische Theorie- und Methodenverständnisse aufzugliedern und in ihre Verwendungskontexte zurückzuverfolgen. ${ }^{33}$

\section{Objekt- und Projektgeschichte}

Aus der Geschichte und Soziologie der Laborwissenschaften kommen Anregungen, die in der Geschichte der Philologien in den vergangenen Jahren mit Interesse aufgegriffen worden sind. ${ }^{34}$ In den Blick gerät neben der Theorie- und Methodenarbeit der große Bereich der eingeübten Routinen, die in einem bloß programmgeschichtlichen Zugang nicht $\mathrm{zu}$ erfassen sind - die buchstäblichen Handgriffe, die auf Erfahrungswissen und Konditionierung, nicht auf kalkulierte Steuerung zurückgehen. ${ }^{35}$ Beginnend in der Philologiegeschichte der Vormoder-

JLTonline (Stand: 05. 09. 2011), URL: http://www.jltonline.de/index.php/reviews/article/view/ 414/1103; ähnliches gilt auch für Tilmann Köppe/Simone Winko: Theorien und Methoden der Literaturwissenschaft. In: Thomas Anz (Hg.): Handbuch Literaturwissenschaft. Bd. 2. Stuttgart/ Weimar 2007, S. 285-372.

32 Inspirierend die quantitative Untersuchung von Anne H. Stevens/Jay Williams: The Footnote, in Theory. In: Critical Inquiry 32 (2006), S. 208-225.

33 Eine Metasuche in der Virtuellen Fachbibliothek Germanistik ergibt unter dem Titelansatz „*theor*“ 7.658 Treffer im Deutschen Literaturarchiv Marbach, 2.084 Treffer im Institut für Deutsche Sprache in Mannheim, 1.749 Treffer im Fachkatalog Germanistik der UB Frankfurt (24. Juli 2012).

34 Wegweisend Hans-Jörg Rheinberger: Experimentalsysteme und epistemische Dinge. Eine Geschichte der Proteinsynthese im Reagenzglas [2001]. Frankfurt/M.: Suhrkamp 2006; Andrew Pickering (Hg.): Science as Practice and Culture. Chicago: University Press 1992; als Klassiker Bruno Latour/Steve Woolgar: Laboratory Life. The Social Construction of Scientific Facts. Beverly Hills: Sage 1979.

35 Mit ausführlichen Forschungshinweisen Carlos Spoerhase/Steffen Martus: Praxeologie der Literaturwissenschaft. In: Geschichte der Germanistik 35/36 (2009), S. 89-96; aus didaktischer Perspektive Oliver Ruf: ,Creative Writing Studies‘ und, angewandter Imperativ: literarisches Schreiben lehren und lernen als Herausforderung einer praxeologischen Philologie. In: 
ne und des 19. Jahrhunderts zeichnet sich, erstens, eine Geschichte der Praktiken $\mathrm{ab}$, die Bibliotheken und Archive nicht nach diskursiven Entwicklungen, sondern nach Verfahrensspuren befragt. Dazu zählen Arbeiten zur Geschichte der Lehre - der Vorlesung, des Seminars, der Hausarbeit - und der nach 1945 und noch einmal nach 1966 umstrukturierten Hochschuldidaktik. ${ }^{36}$ Forschungsbezogene Argumentationsmodi und Durchsetzungsstrategien geraten in den Blick der Kontroversengeschichte. ${ }^{37}$ Werden Fragen und Antwortstrategien, Versprechen und Einlösungsversuche aus praxishistorischer Sicht beleuchtet, dann kann Theorie- und Methodenarbeit in ihrer Prozessualität und Pragmatik, nicht bloß in ihren Ergebnissen erfasst werden. ${ }^{38}$

Wirkendes Wort 59/III (2009), S. 485-501; die Frühneuzeitforschung nimmt mit ihren Studien zur Gelehrsamkeit in praxishistorischer Hinsicht eine Pionierstellung ein, so bei Helmut Zedelmaier/Martin Mulsow (Hg.): Die Praktiken der Gelehrsamkeit in der Frühen Neuzeit. Tübingen: Niemeyer 2001.

36 Holger Dainat: Hochschullehre. In: Thomas Anz (Hg.): Handbuch Literaturwissenschaft. Bd. 3. Stuttgart/Weimar: Metzler 2007, S. 199-208; Heinrich Kaulen: Schule und Hochschule. In: ebd., S. 239-250; Marcel Lepper: Zu welchem Ende sammelt und ediert man Vorlesungen aus Wissenschaftlernachlässen? In: Geschichte der Germanistik 33/34 (2008), S. 48-56; Jörg Schönert: Zur Relevanz des literaturwissenschaftlichen Grundstudiums für disziplinäre Entwicklungen in den Neuphilologien 1970-2010: Impulse, Indikatoren, Imperative. In: Geschichte der Germanistik 37/38 (2010), S. 90-98; Michael Kämper-van den Boogaart/Steffen Martus/Carlos Spoerhase: Entproblematisieren: Überlegungen zur Vermittelbarkeit von Forschungswissen, zur Vermittlung von ,falschem‘ Wissen und zur Funktion

literaturwissenschaftlicher Terminologie. In: Zeitschrift für Germanistik N.F. 21/I (2011), S. 8-24; Schwerpunkt ,Was wäre die Germanistik ohne Lehrerbildung?‘ im Themenheft der Mitteilungen des Deutschen Germanistenverbandes 59/II (2012): Germanistik und Lehrerbildung. Debatten und Positionen. Hg. v. Mark-Georg Dehrmann/Jan Standke. Mit Beiträgen von Katrin Berwanger, Klaus-Michael Bogdal, Jürgen Kaube, S. 118-127.

37 Carlos Spoerhase: Wissenschaftsgeschichte als Konfliktgeschichte. Am Beispiel von Kontroversen in der Literaturtheorie. In: Geschichte der Germanistik 29/30 (2006), S. 17-24; Ralf Klausnitzer/Carlos Spoerhase (Hg.): Kontroversen in der Literaturtheorie, Literaturtheorie in der Kontroverse. Bern u. a.: Lang 2007 (Publikationen zur Zeitschrift für Germanistik, N.F., 19). 38 Carlos Spoerhase: ,Mere reading‘. Über das Versprechen eines ,posthermeneutischen` Verstehens. In: Marcel Lepper/Steffen Siegel/Sophie Wennerscheid (Hg.): Jenseits des Poststrukturalismus? Frankfurt/M.: Lang 2005 S. 15-36; Dirk Werle: Frage und Antwort, Problem und Lösung: zweigliedrige Rekonstruktionskonzepte literaturwissenschaftlicher Ideenhistoriographie. In: Scientia Poetica 13 (2009), S. 255-303; Marcel Lepper: Heuristikgeschichte: ein zweigliedriges Rekonstruktionskonzept. In: ebd., S. 329-338; Jürgen Paul Schwindt (Hg.): Was ist eine philologische Frage? Beiträge zur Erkundung einer theoretischen Einstellung. Frankfurt/M.: Suhrkamp 2009; Anne Bohnenkamp u. a. (Hg.): Konjektur und Krux: Zur Methodenpolitik der Philologie. Göttingen: Wallstein 2010; Remigius Bunia: Das Handwerk in der Theoriebildung. Zu Hermeneutik und Philologie. In: Journal of Literary Theory 5/II (2011), S. 149-162. 
Zur Geschichte von Praktiken tritt, zweitens, eine Geschichte der Objektkonstitution. ${ }^{39}$ In den historischen Disziplinen bedarf es keiner konstruktivistischen Voraussetzungen, um zu zeigen, dass der Gegenstand der Untersuchung nur dann angemessen erfasst werden kann, wenn man die Geschichte seiner Konstituierung und Beobachtung mitdenkt. An die Stelle einer klassischen Editionsgeschichte tritt, wissenschaftshistorisch konzipiert, die Rekonstruktion von Stabilisierungsprozessen: Wie kommen Kanonformationen und Korpora zustande? ${ }^{40}$ Für die Germanistikgeschichte nach 1945 ist das problematische Verhältnis zur literarischen Zeitgeschichte von besonderem Interesse. Wie werden die Texte der verfemten Avantgarden zu modernen Klassikern ${ }^{41}$ Wie kann die Germanistik ein professionelles Verhältnis zur Gegenwartsliteratur aufbauen, das die Option literarischer Rückkopplung einkalkuliert? ${ }^{42}$

39 Wegweisend Lorraine Daston/Peter Galison: Objectivity. Cambridge: MIT Press 2007; Anke te Heesen: Über Gegenstände der Wissenschaft und ihre Sichtbarmachung. In: Zeitschrift für Kulturwissenschaften 1 (2007), S. 95-102; Hans-Jörg Rheinberger: Objekt und Repräsentation. In: Bettina Heintz/Jörg Huber (Hg.): Mit dem Auge denken. Strategien der Sichtbarmachung in wissenschaftlichen und virtuellen Welten. Wien: Springer 2001, S. 55-61; fachpolitisch skeptisch Ulrike Zeuch: Zum Verlust des Gegenstandes in der Literaturtheorie seit 1966. In: Euphorion 95 (2001), S. 101-121.

40 Exemplarisch zur Geschichte der Hölderlin-Forschung nach 1945 Marcel Lepper: Am Quell? Zur Geschichte der Hölderlin-Philologie. In: Geschichte der Germanistik 31/32 (2007), S. 25-33; M.L.: Gegen die ,Naïveté der Wissenschaft‘? Hölderlin-Editoren im Deutschen Literaturarchiv. In: Zeitschrift für Germanistik N.F. 17/II (2007), S. 498-502; Ute Oelmann: Hölderlin Entdeckungen: Studien zur Rezeption. Stuttgart: Württembergische Landesbibliothek 2008; Dieter Burdorf (Hg.): Edition und Interpretation moderner Lyrik seit Hölderlin. Berlin/New York: de Gruyter 2010; Marcel Lepper: Ruhm oder Relevanz? Dichter- und Philologennachlässe im Archiv. In: Stephan Kurz/Michael Rohrwasser/Daniela Strigl (Hg.): Der Dichter und sein Germanist. Symposium in Memoriam Wendelin Schmidt-Dengler. Wien: New Academic Press 2012, S. 64-69.

41 Auswahlweise: Klassiker in finsteren Zeiten 1933-1945. Eine Ausstellung des Deutschen Literaturarchivs. Marbach: Deutsche Schillergesellschaft 1983; Günter Erbe: Die verfemte Moderne. Die Auseinandersetzung mit dem ,Modernismus‘ in Kulturpolitik, Literaturwissenschaft und Literatur der DDR. Opladen: Westdeutscher Verlag 1993; Marcel Lepper/Alexander Nebrig (Hg.): Expressionismus 1960. Themenheft der Mitteilungen des Deutschen Germanistenverbandes. Göttingen: Vandenhoeck \& Ruprecht, 2011.

42 Steffen Martus: ,In der Hölle soll sie braten'. Zur Literatur der Literaturwissenschaft mit einem Seitenblick auf Matthias Polityckis ,Weiberroman' und die Computerphilologie. In: Zeitschrift für Germanistik N.F. 17/I (2007), S. 8-27; als ,locus classicus‘ Walter Hinck: Germanistik als Literaturkritik: zur Gegenwartsliteratur. Frankfurt/M.: Suhrkamp 1983; spezialisiert die Zeitschrift Gegenwartsliteratur: ein germanistisches Jahrbuch. Hg. v. Paul Michael Lützeler. Tübingen: Stauffenburg 1 (2002) ff. 
An dritter Stelle rangiert die Geschichte von Projekten. Hat man unter Großforschung und projektförmiger Forschung im 19. Jahrhundert Wörterbuchunternehmungen und Editionen verzeichnet, so kommen in der zweiten Hälfte des 20. Jahrhunderts große Theorie- und Begriffsunternehmungen hinzu - etwa das Bielefelder Utopieprojekt. ${ }^{43}$ Das Verhältnis von Forschungsplanung und Drittmittelpolitik rückt nicht nur in den Interessenbereich der traditionellen Wissenschaftsgeschichte, sondern auch einer evaluativen Wissenschaftsforschung, die ihre Ergebnisse zurück in die Forschungspolitik spielt. ${ }^{44}$ Dazu zählt auch eine Geschichte der gescheiterten Pläne, der tragischen Heroisierung, der ausgeschalteten Optionen - so wie sie Walter Erhart auf literaturgeschichtlicher Seite exemplarisch für Wolfgang Koeppen rekonstruiert hat. ${ }^{45}$

43 Carlos Spoerhase: Big Humanities. ,Größe‘ und ,Großforschung als Kategorien geisteswissenschaftlicher Selbstbeobachtung. In: Geschichte der Germanistik 37/38 (2010), S. 9-27; über die Konturen einzelner Forschungsprojekte hinaus gehen zeitzeugengestützte Projektgeschichten wie die von Michael Schlott beschriebene: Germanistische Aufklärungsforschung. In: Geschichte der Germanistik 39/40 (2011), S. 104-109.

44 Marcel Lepper: Wissenschaftsplanung und Förderpolitik. Schwerpunkt. Mit Beiträgen von Carlos Spoerhase, Hans-Harald Müller, Sandra Richter, Manuel Braun, Dirk Werle. In:

Geschichte der Germanistik 37/38 (2010), S. 7-59; für die Literaturwissenschaft Eberhard Lämmert: Wissenschaftsgeschichte und Forschungsplanung. In: Walter Müller-Seidel u. a. (Hg.): Historizität in Sprach- und Literaturwissenschaft. Vorträge und Berichte der Stuttgarter Germanistentagung 1972. München: Fink 1974, S. 663-685; Sandra Pott: Leitbegriffe für die Wissenschaftsförderung. Zum Zusammenhang von Wissenschaftspolitik und disziplinärer Entwicklung in der germanistischen Literaturwissenschaft seit 1983. In: Jörg Schönert (Hg.): Literaturwissenschaft und Wissenschaftsforschung. Stuttgart/Weimar: Metzler 2000, S. 551572; Thomas Wiemer: Forschungsförderung. In: Thomas Anz (Hg.): Handbuch Literaturwissenschaft. Bd. 3. Stuttgart/Weimar: Metzler 2007, S. 223-227; für die Linguistik Andreas Gardt: Forschungsförderung in der Sprachwissenschaft. In: Geschichte der Germanistik 39/40 (2011), S.63-69; für die Mediävistik das Themenheft Germanistische Mediävistik: Forschungsförderung. In: Mitteilungen des Deutschen Germanisten-Verbandes 33/ I (1986), S. 2-43; für die Didaktik Bernd Dammann: Forschungsförderung und Lehrerausbildung: eine wissenschaftssoziologische Studie zu Selbstverständnis und Funktion der Geisteswissenschaften im Horizont wissenschaftsorganisatorischer Strukturveränderungen des Forschungs- und Ausbildungssystems. Bremen [masch.] 1975.

45 Walter Erhart: Wolfgang Koeppen. Das Scheitern moderner Literatur. Konstanz: Konstanz University Press 2012; auf wissenschaftshistorischer Seite bietet Jürgen Fohrmann ein Makromodell für eine Geschichte, die im 18. und 19. Jahrhundert nicht nur Projekterfolg, sondern auch Projektscheitern rekonstruieren kann: Das Projekt der deutschen Literaturgeschichte. Entstehung und Scheitern einer nationalen Poesiebeschreibung zwischen Humanismus und Deutschem Kaiserreich. Stuttgart/Weimar: Metzler 1989. 


\section{Institutionengeschichte}

Eine Geschichte von Praktiken, Objekten und Projekten bietet ein wirkungsvolles Korrektiv zur Institutionengeschichte, die häufig Gefahr läuft, die Selbstbeschreibung von Fächern und Einrichtungen alternativlos nachzuerzählen oder abzuwickeln. ${ }^{46}$ Ein Disziplinenbegriff, wie ihn die an Rudolf Stichweh anschließende Forschung verwenden kann, sorgt für einen historisch reflektierten Umgang mit dem Fachterminus der Germanistik. ${ }^{47}$ Institutionengeschichtliche Studien für die Zeit nach 1945 widmen sich, erstens, nicht bloß der Entwicklung von Einzelinstituten, ${ }^{48}$ sondern der Wechselwirkung von Infrastruktur- und Methodenentwicklung - in besonderer Weise den Plänen, Prozessen und Folgen der institutionellen Expansion, dem Ausbau der Germanistik zu einem Massenfach. ${ }^{49}$

Relevant ist, zweitens, der internationale, komparatistische Zugang - die Historisierung des problematischen Begriffs der „Auslandsgermanistik“, die Re-

46 Als Klassiker dagegen Timothy Lenoir: Instituting Science: The Cultural Production of Scientific Disciplines. Stanford: Stanford University Press 1997.

47 Rudolf Stichweh: Zur Entstehung des modernen Systems wissenschaftlicher Disziplinen. Physik in Deutschland 1740-1890. Frankfurt/M.: Suhrkamp 1984; Jürgen Fohrmann/Wilhelm Voßkamp (Hg.): Von der gelehrten zur disziplinären Gemeinschaft. Stuttgart/Weimar: Metzler 1987 (Sonderheft der DVjs 61); anders akzentuiert Lutz Danneberg u. a. (Hg.): Stil, Schule, Disziplin. Analyse und Erprobung von Konzepten wissenschaftsgeschichtlicher Rekonstruktion. Frankfurt/M.: Lang 2005.

48 Häufig eingebettet in Gründungsgeschichten, so Myriam Richter (Hg.): 100 Jahre Germanistik in Hamburg: Traditionen und Perspektiven. Hamburg: Reimer 2011; 200 Jahre Berliner Germanistik. Schwerpunktthema. In: Zeitschrift für Germanistik N.F. 20/I-II (2010),; Andreas Pilger: Germanistik an der Universität Münster: von den Anfängen um 1800 bis in die Zeit der frühen Bundesrepublik. Heidelberg: Synchron 2004; Georg Machnik (Hg.): Germanistik in Jena: Reden aus Anlaß des 70. Geburtstags von Heinz Mettke, 10. Januar 1995. Jena: Friedrich-Schiller-Univ., 1996.

49 Henry J. Schmidt: What is Oppositional Criticism? Politics and German Literary Criticism from Fascism to the Cold War. In: Monatshefte 79 (1987), S. 292-307; Christoph König: Wissen, Werte, Institutionen. Zum Marbacher Symposion über Kontinuität und Diskontinuität in der neueren deutschen Literaturwissenschaft nach 1945. In: Jahrbuch der Deutschen Schillergesellschaft 38 (1994), S. 379-403; Rainer Rosenberg/Inge Münz-Koenen/Petra Boden (Hg.): Der Geist der Unruhe. 1968 im Vergleich: Wissenschaft - Literatur - Medien. Berlin: Akademie 2000; unter den älteren Beiträgen Walter Müller-Seidel: Die Erforschung der deutschen Literatur: Zur Situation in einem sogenannten Massenfach. In: Hellmut Flashar (Hg.): Geisteswissenschaft als Aufgabe: kulturpolitische Perspektiven und Aspekte. Berlin/New York: de Gruyter 1978, S. 137-152; Burghard Rieger: Von den Ansichten einer künftigen Germanistik zu den Germanisten ohne Zukunft? Über alternative Tätigkeitsfelder für Philologen in einer veränderten kommunikativen Infrastruktur unserer Gesellschaft. In: Der Deutschunterricht 35/IV (1983), S. 59-75. 
konstruktion von Differenzen im globalen Fachkontext. ${ }^{50}$ Zweifellos spielen OstWest-Vergleiche eine wesentliche Rolle,$^{51}$ ebenso die unterschiedlichen Entwicklungen in der Schweiz und in Österreich. ${ }^{52}$ Wissenschaftspolitisch relevant wird eine germanistische Institutionengeschichte unter Gefährdungsbedingungen.

50 Michael Braun: Vielheit in der Einheit der Germanistik weltweit (XII. Kongress der IVG in Warschau v. 30. 07.-07. 08. 2010). In: Zeitschrift für Germanistik N.F. 21/II (2011), S. 376-378; Marcel Lepper: Welche Auslandsgermanistik? In: Jahrbuch der Deutschen Schillergesellschaft 52 (2008), S. 14-17; M.L.: Welche Auslandsgermanistik? Einladung zur zweiten Diskussionsrunde. In: Jahrbuch der Deutschen Schillergesellschaft 53 (2009), S. 345; mit Beiträgen von Michel Espagne, Rüdiger Görner, Walter F. Veit, Friedrich Voit, Liliane Weissberg, S. 346-378; Welche Auslandsgermanistik? Bilanz der zweiten Diskussionsrunde. In: Jahrbuch der Deutschen Schillergesellschaft 53 (2010), S. 515-516; mit Beiträgen von Christoph Bartmann, Maria Carolina Foi, Christine Ivanovic und Paolo Astor Soethe, S. 517-536; Franciszek Grucza (Hg.): Deutsch und Auslandsgermanistik in Mitteleuropa. Geschichte, Stand, Ausblicke. Dokumentation einer internationalen Konferenz 10.-12. Oktober 1996. Warszawa: Graf-Punkt 1998.

51 Zur DDR-Germanistik auswahlweise Reinhard Hahn: Gerhard Scholz und sein Kreis. Zur Schulenbildung in der Germanistik der DDR. In: Jahrbuch für Universitätsgeschichte 12 (2009), S. 133-156; Jens Saadhoff: Germanistik in der DDR. Literaturwissenschaft zwischen gesellschaftlichem Auftrag und disziplinärer Eigenlogik. Heidelberg: Synchron 2007; Petra Boden/Dorothea Böck (Hg.): Modernisierung ohne Moderne. Das Zentralinstitut für Literaturgeschichte an der Akademie der Wissenschaften der DDR (1969-1991). Heidelberg: Winter 2004; Wolfhart Henckmann/Gunter Schandera (Hg.): Ästhetische Theorie in der DDR 1949 bis 1990. Beiträge zu ihrer Geschichte. Berlin: Berlin Verlag 2001; Dagmar Ende/Mandy Funke: Zum Verhältnis von Kontinuität und Diskontinuität in der Literaturwissenschaft der DDR in den 1980er Jahren. In: Jörg Schönert (Hg.): Literaturwissenschaft und Wissenschaftsforschung. Stuttgart/Weimar 2000, S. 399-429; Joachim Lehmann: Die blinde Wissenschaft. Realismus und Realität in der Literaturwissenschaft der DDR. Würzburg: Königshausen und Neumann 1995; Gunter Schandera: Autoritäres System - Autorität der Wissenschaft? Bemerkungen zur Rezeptionsästhetik in der DDR. In: Euphorion 92/III (1998), S. 361-374; Rainer Rosenberg: Zur Begründung der marxistischen Literaturwissenschaft der DDR. In: R.R./Petra Boden (Hg.): Deutsche Literaturwissenschaft 1945-1965. Fallstudien zu Institutionen, Diskursen, Personen. Berlin: Akademie 1997, S. 203-240; zur Evaluationsgeschichte Heinz Ludwig Arnold/Frauke Meyer Gosau (Hg.): Die Abwicklung der DDR. Göttingen: Wallstein 1992; Rainer Rosenberg: Zur Geschichte der Literaturwissenschaft in der DDR. In: Zeitschrift für Germanistik N.F. 1 (1991), S. 247-256.

52 Dazu Ursula Amrein: Diskurs der Mitte: antimoderne Dichtungstheorien in der Schweizer Germanistik vor und nach 1945. In: Internationales Archiv für Sozialgeschichte der deutschen Literatur 26/I (2001), S. 36-57; Corinna Caduff/Michael Gamper (Hg.): Schreiben gegen die Moderne: Beiträge zu einer kritischen Fachgeschichte der Germanistik in der Schweiz. Zürich: Chronos 2001; Wendelin Schmidt-Dengler (Hg.): Probleme und Methoden der Literaturgeschichtsschreibung in Österreich und in der Schweiz. Wien: Praesens 1997; W.S.-D.: (Hg.): Germanistik im Spannungsfeld zwischen Philologie und Kulturwissenschaft. Wien: Praesens 1999. 
Germanistik in Osteuropa, West- und Nordafrika, im Nahen Osten, in Ostasien und Südamerika wurde lange Zeit rein aus förderpolitischer Perspektive wahrgenommen. Eine Geschichte von Praktiken ermöglicht überraschende Befunde, die eine oft allzu stark westeuropäisch geführte Debatte anregen können. ${ }^{53}$ Sind wie in den USA und in Westeuropa germanistische Institute von institutionellen Einschnitten bedroht, so erhält die Wissenschaftsgeschichte standortpolitisches Gewicht. ${ }^{54}$

Schließlich, drittens, rückt die Geschichte von Institutionen in den Blick, die quer zu den Instituten liegen: die Rekonstruktion der Tätigkeit von Verbänden (DGV, IVG), Forschergruppen, Kommissionen, Vereinen, Gesellschaften, Zeitschriften, Verlagen - die Arbeit von wissenschaftlichen Schulen und Netzwerken unter kompetitiven Bedingungen. ${ }^{55}$ Germanistische Redaktionsarchive und Verbandsakten, wie sie etwa im Deutschen Literaturarchiv Marbach für die Forschung

53 In globaler Hinsicht etwa Georg M. Gugelberger: Germanistik, the Canon, and Third World Literature. In: Monatshefte 83 (1991), S. 45-58; Teruaki Takahashi: Japanische Germanistik auf dem Weg zu einer kontrastiven Kulturkomparatistik: Geschichte, Theorie, Fallstudien. München: Fink 2006; Son Seongho: Die Geschichte der germanistischen Sprachwissenschaft in Korea. In: Zeitschrift für Literaturwissenschaft und Linguistik 32 (2002), S. 97-109; Sabine Wilke: Zwanzig Jahre Germanistik postkolonial. In: Monatshefte 103/III (2011), S. 425-439.

54 Traditionsreich ist insbesondere die fachpolitische Reflexion in den USA, so bei Otto W. Johnston: Die Problematik der Germanistik in den USA. In: Jahrbuch für internationale Germanistik 15/I (1983), S.132-149; Frank Trommler (Hg.): Germanistik in den USA. Neue Entwicklungen und Methoden. Opladen: Westdt. Verlag 1989; Paul Michael Lützeler:

Literaturwissenschaft - German Studies - Interkulturelle Germanistik: Zur ,Krise“ des Faches Deutsch in den USA. In: Mitteilungen des Deutschen Germanistenverbandes 37/I (1990),

S. 31-37; Germanistik in den USA. Themenschwerpunkt. In: Weimarer Beiträge 39/III (1993),

S. 325-477; John A. McCarthy (Hg.): The Future of Germanistik in the USA: Changing our Prospects: Nashville: Vanderbilt University Press 1996; Thomas W. Kniesche: Germanistik im Spannungsfeld von Markt und Öffentlichkeit: Beobachtungen aus den USA. In: Jahrbuch der Deutschen Schillergesellschaft 47 (2003), S. 410-414.

55 Paradigmatisch an einem Modellfall der Jahrhundertwende Hans-Harald Müller, Mirko Nottscheid (Hg.): Wissenschaft ohne Universität, Forschung ohne Staat. Die Berliner Gesellschaft für deutsche Literatur (1888-1938). Berlin/New York: de Gruyter 2011; Arnd Beise: Gesellschaften. In: Thomas Anz (Hg.): Handbuch Literaturwissenschaft. Bd. 3. Stuttgart/ Weimar: Metzler 2007, S. 218-222; Wolfgang Adam: Die Zeitschrift Euphorion in den Jahren 1950-1970. In: Petra Boden/Rainer Rosenberg (Hg.): Deutsche Literaturwissenschaft. 1945-1965. Fallstudien zu Institutionen, Diskursen, Personen. Berlin: Akademie Verlag 1997, S. 241-260; Gunter Schandera: Die Weimarer Beiträge zwischen 1955 und 1961. Eine Zeitschrift auf dem Weg zum zentralen Organ der marxistischen Literaturwissenschaft in der DDR? In: ebd., S. 261-332; Christiane Kussin (Hg.): Literarische Gesellschaften in Deutschland. Berlin: Aufbau 1995; Klaus Röther: Die Germanistenverbände und ihre Tagungen. Ein Beitrag zur germanistischen Organisations- und Wissenschaftsgeschichte. Köln: Pahl 1980. 
erschlossen werden, bieten dafür eine Grundlage - so die Bestände der Deutschen Vierteljahrsschrift für Literaturwissenschaft und Geistesgeschichte (DVjs), des Deutschen Germanistenverbands (DGV), der Deutschen Gesellschaft für Allgemeine und Vergleichende Literaturwissenschaft (DGAVL), der Zeitschrift Germanistik, der Heinrich-von-Kleist-Gesellschaft, der Internationalen Vereinigung für Germanische Sprach- und Literaturwissenschaft (IVG), des Internationalen Archivs für Sozialgeschichte der deutschen Literatur (IASL), des Reallexikons der deutschen Literaturgeschichte, des Reallexikons der deutschen Literaturwissenschaft (RL), des Kritischen Lexikons zur deutschsprachigen Gegenwartsliteratur (KLG). ${ }^{56}$

\section{Personengeschichte}

Seit den frühneuzeitlichen Gelehrtenlexika bereitet die Personengeschichte einen erheblichen Grundlagenanteil der Wissenschaftsgeschichtsschreibung vor. ${ }^{57}$ Müssen sich Problemansätze quer zum Provenienzprinzip der Archive verhalten, kann die personengeschichtliche Wissenschaftsgeschichte sich auf die archivarische Gliederungsstruktur beziehen. ${ }^{58}$ Sie ist damit freilich, wenn sie sich in der Zeitgeschichte bewegt, an die Schwierigkeiten des Überlieferungsprozesses und der Archivierungsentscheidungen gebunden. Relevant ist, erstens, der Schutz lebender Personen, der im vorliegenden Fall eine Benutzung unpublizierter Bestände einschränkt, die Material von, an und über solche enthalten. Bestände und Bestandsteile von Germanistinnen und Germanisten der 1920er Jahrgänge, die ihre Karrieren Mitte der 1950er Jahre beginnen, erst recht Archivalien zu den in den 1930er und 1940er Jahren geborenen Fachkollegen, sind nur mit Einwilligung der Betroffenen benutzbar. Das Persönlichkeitsrecht und der Persönlichkeitsschutz, abgeleitet aus dem Grundgesetz (Art. 1 in Verbindung mit Art. 2), Landesarchivgesetze und Benutzungsordnungen der einzelnen Archive setzen der jüngsten Wissenschaftsgeschichte wenn nicht einheitliche, so in jedem Fall erhebliche Grenzen. Zweitens spielt das Urheberrecht, anders als in der gegen-

56 Bestandsbeschreibungen gehen aus dem OPAC Kallías hervor: http://www.dla-marbach. de/opac_kallias/index.html; darüber hinaus finden sich zahlreiche bedeutende Bestände in Universitätsarchiven, Universitätsbibliotheken und, sofern erhalten, in den Altablagen der wissenschaftlichen Verlage.

57 Bernd Hamacher: Historische Kernbereiche der Germanistik im Lichte der Fachentwicklung. Poetik - Biographik - Lexikographie. In: Deutsche Vierteljahrsschrift für Literaturwissenschaft und Geistesgeschichte 81/IV (2007), S. 638-656.

58 Christoph König/Hans-Harald Müller/Werner Röcke (Hg.): Wissenschaftsgeschichte der Germanistik in Porträts. Berlin/New York: de Gruyter 2000, darin u. a. Gesa Dane zu Käte Hamburger, S. 189-198. 
wärtigen Forschung zum 19. und frühen 20. Jahrhundert, eine erhebliche Rolle es erlischt 70 Jahre nach dem Tod des Urhebers (§ 64 UrhG). Ist im Jahr 2012 unpubliziertes Material nur von den Autoren frei zitierbar, die vor 1942 verstorben sind, so fällt der komplette Bereich der Wissenschaftsgeschichte nach 1945 noch für die nächsten Jahrzehnte unter urheberrechtliche Regelungen - im wohlbegründeten Sinne wissenschaftlicher Autoren und ihre Erben, zugleich mit allen Schwierigkeiten für den freien Materialumgang.

Fragen ergeben sich, drittens, aus der Überlieferungssituation - der historisch einmaligen Menge überlieferten Materials einerseits, der noch laufenden Archivierungsprozesse andererseits. Wer Zeitgeschichte der Wissenschaften schreibt, muss sich auf ein hohes Maß an Archivbewusstsein der Bestandsbildner gefasst machen - auf Selbstzensur, Inszenierung, Memorialpolitik, Sammlungs- und Ablagepraktiken als Teil der wissenschaftlichen Tätigkeit. ${ }^{59}$ Große Teile des wissenschaftlichen Archivmaterials für die Zeit nach 1945 befinden sich noch in Privatbesitz oder in den Ablagen universitärer Institute, so dass übergreifende Studien mit erheblichen Hindernissen konfrontiert sind. Die Herausforderung des Umgangs mit Zeitzeugen, die die Zeitgeschichte kennt, ist, viertens, in der Wissenschaftsgeschichte von besonderer Bedeutung. Verfahren der Oral history, die ergänzend zu Archivstudien eingesetzt werden, erhalten den Status von Experteninterviews. ${ }^{60}$ Schließlich bringen, fünftens, der quantitative Ausbau, die internationalen Ausgründungen und die gesteigerte Binnendifferenzierung der Ger-

59 Marcel Lepper: Zettelwelt, Denklabor. Oder: Was Wissenschaftler hinterlassen. Eine Sichtung. In: Quarto 33/34 (2011), S. 27-33; zur Memorialpolitik gehören auch die Festschriften, die im Zeitraum nach 1945 keineswegs aus der Mode kommen: Der Katalog des Deutschen Literaturarchivs Marbach verzeichnet allein für den Publikationszeitraum 2000-2012 eine Zahl von 651 Festschriften, darunter ein großer Anteil germanistischer Publikationen. 60 Eva M. McMahan/Kim Lacy Rogers: Interactive Oral History Interviewing. London: Routledge 2011; Donald A. Ritchie: The Oxford Handbook of Oral History. Oxford: Oxford University Press 2011; Dora Imhof (Hg.): Interviews. Oral History in Kunstwissenschaft und Kunst. München: Schreiber 2010; Astrid Erll: Biographie und Wissen. In: Christian Klein (Hg.): Handbuch Biographie. Methoden, Traditionen, Theorien. Stuttgart/Weimar: Metzler 2009, S. 71-86; Almut Leh/Lutz Niethammer (Hg.): Kritische Erfahrungsgeschichte und grenzüberschreitende Zusammenarbeit. The Networks of Oral History. Leverkusen: Budrich 2007 (BIOS Sonderheft); Hans J. Markowitsch/Harald Welzer (Hg.): Das autobiografische Gedächtnis. Hirnorganische Grundlagen und biosoziale Entwicklung. Stuttgart: Klett 2005; Anna Green/Megan Hutching (Hg.): Remembering. Writing Oral History. Auckland: Auckland University Press 2004; Konrad Jarausch/Martin Sabrow (Hg.): Verletztes Gedächtnis. Erinnerungskultur und Zeitgeschichte im Konflikt. Frankfurt/M./New York: Campus 2002; Hans J. Markowitsch: Die Erinnerung von Zeitzeugen aus der Sicht der Gedächtnisforschung, in: BIOS. Zeitschrift für Biographieforschung und Oral History 13 (2000), S. 30-50; Harald Welzer: Das Interview als Artefakt. Zur Kritik der Zeitzeugenforschung. In: BIOS 13 (2000), S. 51-63; Wolfgang Kraushaar: 
manistik quantitative Probleme mit sich, die weder mit zentralistischen noch dezentralen Archivstrategien flächendeckend zu lösen sind. ${ }^{61}$ Die nie zuvor erreichte Zahl forschender und lehrender Germanistinnen und Germanisten, die präzedenzlose Menge germanistischer Publikationen steht einer vergleichsweise geringfügigen Zahl zugänglicher Germanistennachlässe gegenüber - welche erwerbenden, erschließenden und bestandserhaltenen Anstrengungen international auch immer unternommen werden. Zeichnen sich die archivierten Bestände durch großen Umfang und hohe Überlieferungsdichte aus, so liegt in der Asymmetrie zwischen Bibliothek und Archiv die eigentliche Schwierigkeit, auf die sich die Wissenschaftsgeschichte einstellen muss. ${ }^{62}$

Welche Fragen die Provenienzheuristik der personengeschichtlichen Forschung mit sich bringt, lässt sich am Deutschen Literaturarchiv Marbach zeigen der gegenwärtig größten Institution zur Sammlung und Erschließung von Germanistenbeständen. In Marbach liegen aus dem Untersuchungszeitraum 1945-2012 u. a. die Vor-, Nach- und Teilnachlässe von Erwin Ackerknecht (1880-1960), Bruno Adler (1889-1968), Richard Alewyn (1902-1979), Beda Allemann (19261991), Heinz Ludwig Arnold (1940-2011), Dieter Bassermann (1887-1955), Wilhelm Bausinger (1935-1964), Adolf Beck (1906-1981), Carl Friedrich Wilhelm Behl (1889-1968), Friedrich Beißner (1905-1977), Richard Benz (1884-1966), Eduard Berend (1883-1973), Ernst Bertram (1884-1957), Bernhard Blume (1901-1978), Lieselotte Blumenthal (1906-1992), Paul Böckmann (1899-1987), Helmut de Boor (1891-1976), Johannes Jacobus Braakenburg (1923-1988), Herbert Cysarz (18961985), Claude David (1913-1999), Horst Denkler (geb. 1935), Jörg Drews (19382009), Adalbert Elschenbroich (1914-1989), Hildegard Emmel (1911-1996), Erika Essen (1914-1986), Erich Fausel (1904-1963), Paul Fechter (1880-1958), Hanns Fischer (1928-1968), Leonard Wilson Forster (1913-1997), Herbert A. Frenzel (1908-1995), Gerhard Fricke (1901-1980), Adolf Frisé (1910-2003), Rainer Gruenter (1918-1993), Käte Hamburger (1896-1992), Walfried Hartinger (1938-2008), Hans-Egon Hass (1916-1969), Walter Haug (1927-2008), Erich Heller (1911-1990),

Der Zeitzeuge als Feind des Historikers? Neuerscheinungen zur 68er-Bewegung. In: Mittelweg 36 (1999), S. 49-72.

61 Entsprechendes gilt für die Lexikographie: Das Germanistenverzeichnis, zuerst in Printform erschienen, seit 1998 digital zur Verfügung gestellt, bietet mit mehr als 4.800 Datensätzen aus über 50 Ländern eine nützliche Hilfestellung, bleibt freilich auf die selektive Selbstauskunft angewiesen. Zwischen dem Terminus ante quem des Internationalen Germanistenlexikons und den ersten Einträgen lässt die digitale Datenbank, deren Vorteil in der kontinuierlichen Aktualisierbarkeit besteht, eine erhebliche Lücke.

62 Zur Binnendifferenzierung des großen Fachs etwa die feingliedrige Systematik der Bibliographie der deutschen Sprach- und Literaturwissenschaft (BDSL) - BDSL Online mit rund 366.000 Titeldaten für den Zeitraum 1985-2011. 
Otto Heuschele (1900-1996), Dorothea Hölscher-Lohmeyer (1913-2008), Klaus Günther Just (1923-1977), Hans Kaufmann (1926-2000), Wolfgang Kayser (19061960), Alfred Kelletat (1916-1995), Friedrich A. Kittler (1943-2011), Alfred von Klement (1889-1957), Paul Kluckhohn (1886-1957), Helmut Kreuzer (1927-2004), Hugo Kuhn (1909-1978), Artur Kutscher (1878-1960), Eberhard Lämmert (geb. 1924), Victor Lange (1908-1996), August Langen (1907-1983), Edgar Lohner (1919-1975), Hans-Joachim Mähl (1923-2001), Fritz Martini (1909-1991), Gert Mattenklott (1942-2009), Harry Maync (1874-1947), Herbert Meyer (1908-1992), Herman Meyer (1911-1993), Werner Milch (1903-1950), Georg Minde-Pouet (18711950), Robert Minder (1902-1980), Werner Mittenzwei (geb. 1927), Günther Müller (1890-1957), Walter Müller-Seidel (1918-2010), Josef Nadler (1884-1963), Hans Naumann (1886-1951), Adolf Nutzhorn (1890-1918), Norbert Oellers (geb. 1936), Wolfgang Paulsen (1910-1998), Johannes Pfeiffer (1902-1970), Heinz Politzer (1910-1978), Hermann Pongs (1889-1979), Wolfgang Preisendanz (1920-2007), Ulrich Pretzel (1898-1981), Hans Pyritz (1905-1958), Fritz Rahn (1891-1964), Walther Rehm (1901-1963), Heinz Ritter (1902-1994), Horst Rüdiger (1908-1984), Erich Ruprecht (1906-1997), Dietrich Eberhard Sattler (geb. 1939), Arno Schirokauer (1899-1954), Wilhelm Schneider (1885-1979), Günter Schulz (19061985), Egon Schwarz (geb. 1922), Hans Schneider/Schwerte (1909-1999), Helmut Sembdner (1914-1997), Heinz Stanescu (1921-1994), Herbert Steiner (1892-1966), Paul Stöcklein (1909-1992), Gerhard Storz (1898-1983), Ingrid StrohschneiderKohrs (geb. 1922), Elida Maria Szarota (1904-1994), Peter Szondi (1929-1971), Claus Träger (1927-2005), Erich Trunz (1905-2001), Fritz Tschirch (1901-1975), Karl Viëtor (1892-1951), Werner Vordtriede (1915-1985), Kurt Wais (1907-1995), Max Wehrli (1909-1998), Günther Weydt (1906-2000), Elsbeth Wolffheim (19342002), Hans Wolffheim (1904-1973), Alfred Zastrau (1906-1981), Bernhard Zeller (1919-2008), Hans Zeller (geb. 1926), Klaus Ziegler (1908-1978), Ernst Zinn (19101990). ${ }^{63}$ Deutlich wird nicht bloß, wie eine Sammlung mit ihren Zentren und Peripherien entlang der Erwerbungsgeschichte über Relevanzentscheidungen Auskunft gibt, sondern auch, jenseits der Kriterien und Kontingenzen, wie vielfältig das Feld germanistischer Tätigkeit trotz der Professionalisierungs- und Institutionalisierungsschübe des 20. Jahrhunderts bleibt - in Universitäten und Akademien, aber auch in Archiven und Bibliotheken, ausgeübt von Beamten und Privatgelehrten, von Forschern und Kennern, von Wissenschaftspolitikern und

63 Details unter http://www.dla-marbach.de/?id=51831; Neuerwerbungen werden jährlich ausführlich in der Zeitschrift Geschichte der Germanistik besprochen; zur Reflexion der Sammlungskriterien http://www.wissenschaftsrat.de/download/archiv/1243-11.pdf (zuletzt eingesehen am 26. Juli 2012). 
Dichterphilologen, von Journalisten und Intellektuellen. ${ }^{64}$ Wertvoll werden Einzelbestände und personengeschichtliche Beiträge dort, wo sie quer zur Provenienzlogik einsehbar und befragbar sind - auf übergeordnete theorie- und methodengeschichtliche, objekt- und projektgeschichtliche, ebenso auf generationen- und genderpolitische Anliegen hin. ${ }^{65}$

\section{Wege}

Hat der Konflikt zwischen der Entgrenzung des Sprach- und Literaturbegriffs einerseits, der ,Re-Philologisierung ' andererseits seinen eigenen fachhistorischen Index? Ein integrativer, nicht integristischer Philologiebegriff, wie er seit mehr als einem Jahrzehnt die internationalen Debatten belebt, hat die Fronten längst verschoben. Er trägt dazu bei, die einzelnen Philologien und die sprach- und literaturwissenschaftlichen Querschnitte, die Binnenkonflikte zu entpartikularisieren. ${ }^{66}$ Die Philologiegeschichte tut gut daran, die Furcht der Fächer vor der antiquarischen Desorientierung wie vor der historischen Arroganz ernst zu nehmen. Sie hat ihren Ort im Kern der philologischen Fächer selbst - als disziplinäre Grundfertigkeit der kritischen Selbstbeschreibung, als Reflexion über die Fächer, ihre Genesen, ihre Gegenstände und Werkzeuge, im Bewusstsein ihres Wirkungspotentials in evaluativen wie in steuernden Diskursen. ${ }^{67}$

64 Dazu ausführlich Christoph König: Einleitung (Anm. 4), S. XV.

65 Walter Erhart: Generationen - zum Gebrauch eines alten Begriffs für die jüngste Geschichte der Literaturwissenschaft. In: Jörg Schönert (Hg.): Literaturwissenschaft und Wissenschaftsforschung. Stuttgart/Weimar: Metzler 2000, S. 77-100; W.E.: The Gender of Philology. A Genealogy of Germanistik. In: Miriam Kauko u. a. (Hg.): Gendered Academia. Wissenschaft und Geschlechterdifferenz 1890-1945. Göttingen: Wallstein 2005, S. 41-64. 66 Auswahlweise Hans Ulrich Gumbrecht: Die Macht der Philologie [2002]. Frankfurt/M.: Suhrkamp 2003; Ottmar Ette: ÜberLebenswissen. Die Aufgabe der Philologie. Berlin 2004; Peter-André Alt: Die Verheißungen der Philologie. Göttingen: Wallstein 2007; Werner Hamacher: Für - Die Philologie. Basel 2009; Christoph König (Hg.): Das Potential europäischer Philologien. Geschichte, Leistung, Funktion. Göttingen: Wallstein 2009; Sheldon Pollock: Future Philology? The Fate of a Soft Science in a Hard World. In: Critical Inquiry 35/IV (2009), S. 931-961; Nikolaus Wegmann: How to do Things with Philology [Vortrag an der Columbia University, 24. Februar 2012, ungedrucktes Manuskript]; Marcel Lepper: Philologie. Zur Einführung. Hamburg: Junius 2012.

67 Holger Dainat: ,... die Dinge selbst in Bewegung setzen‘. Fachentwicklung und Selbstreflexion in der bundesrepublikanischen Germanistik seit den sechziger Jahren. In: Johannes Janota (Hg.): Kultureller Wandel und die Germanistik der Bundesrepublik. Vorträge des Augsburger Germanistentages. Bd. 2: Germanistik und Deutschunterricht im historischen Wandel. Tübingen: Niemeyer 1993, S. 207-216. 
Die Gefahr der Epochenkante im Blick: Eine Zeitgeschichte historischer Disziplinen bleibt mutlos, wenn sie keinen Begriff von der wissenschaftlichen Gegenwart, keine eigenen Vorstellungen zumindest von der wissenschaftshistorischen Zukunft entwickelt. Germanistikgeschichte für die Zeit nach 1945 kann sich nicht leisten, flächige Annahmen über das Fach zugrunde zulegen - sie muss, erstens, im besten Sinne philologischer, das heißt auch komparativer werden. ${ }^{68}$ Tiefenschärfe kann sie nur aufgrund effizienter Verfahren gewinnen. Insofern muss sie, zweitens, mit größeren Korpora umgehen, digitale Werkzeuge in bibliometrischen, korpuslinguistischen und begriffsgeschichtlichen Untersuchungen verfeinern. ${ }^{69}$ Sie muss, drittens, globaler denken und in engmaschigeren Netzen, gegenwärtiger und mit längerem Gedächtnis - im Rahmen einer künftigen Geschichte der Philologien. ${ }^{70}$

68 In Archiven wie dem Deutschen Literaturarchiv Marbach treten deshalb neben Germanistennachlässe im Zeitraum nach 1945 bedeutende nichtgermanistische Philologenbestände, so die Vor- und Nachlässe von Erich Auerbach (1892-1957), Wolfgang Iser (1926-2007), Hans Robert Jauß (1921-1997), Manfred Naumann (geb. 1925), Eva RechelMertens (1895-1981), Lea Ritter Santini (1928-2008), Walter Rüegg (geb. 1918), Robert Weimann (geb. 1928).

69 Fotis Jannidis: Methoden der computergestützten Textanalyse. In: Vera Nünning (Hg.): Methoden der literatur- und kulturwissenschaftlichen Textanalyse. Stuttgart 2010, S. 109-132; Heike Neuroth/Fotis Jannidis/Andrea Rapp/Felix Lohmeier: Virtuelle Forschungsumgebungen für e-Humanities. Maßnahmen zur optimalen Unterstützung von Forschungsprozessen in den Geisteswissenschaften. In: Bibliothek. Forschung und Praxis 33/II (2009), S. 161-169. 70 „Disciplines can no longer be merely particular forms of knowledge that pass as general under the mask of science; instead, they must emerge from a new global, and preferably globally comparative, episteme and seek global, and preferably globally comparative, knowledge“; Pollock: Future Philology? (Anm. 66), S. 948; Zukunftsphilologie. Revisiting the Canons of Textual Scholarship (http://www.forum-transregionale-studien.de/

zukunftsphilologie/ [2010]; zuletzt eingesehen am 2. Juni 2012). 